\title{
Unconventional phase III of high-pressure solid hydrogen
}

\author{
Sam Azadi* \\ Department of Physics, King's College London, Strand, London WC2R 2L, United Kingdom \\ and Department of Physics, Imperial College London, Exhibition Road, London SW7 2AZ, United Kingdom \\ Thomas D. Kühne \\ Chair of Theoretical Chemistry and Paderborn Center for Parallel Computing, University of Paderborn, Warburger Str. 100, \\ D-33098 Paderborn, Germany
}

(Received 19 June 2019; revised manuscript received 27 August 2019; published 2 October 2019)

\begin{abstract}
We reassess the phase diagram of high-pressure solid hydrogen using mean-field and many-body wave function-based approaches to determine the nature of phase III of solid hydrogen. To discover the best candidates for phase III, density functional theory calculations within the metageneralized gradient approximation by means of the strongly constrained and appropriately normed (SCAN) semilocal density functional are employed. We study 11 molecular structures with different symmetries, which are the most competitive phases, within the pressure range of 100 to $500 \mathrm{GPa}$. The SCAN phase diagram predicts that the $C 2 / c-24$ and $P 6_{1} 22-36$ structures are the best candidates for phase III with an energy difference of less than $1 \mathrm{meV} /$ atom. To verify the stability of the competitive insulator structures of $C 2 / c-24$ and $P 6_{1} 22-36$, we apply the diffusion Monte Carlo (DMC) method to optimize the percentage $\alpha$ of exact exchange in the trial many-body wave function. We found that the optimized $\alpha$ equals $40 \%$ and denote the corresponding exchange and correlation functional as PBE1. The energy gain with respect to the well-known hybrid functional PBE0, where $\alpha=25 \%$, varies with density and structure. The PBE1-DMC enthalpy-pressure phase diagram predicts that the $P 6_{1} 22-36$ structure is stable up to $210 \mathrm{GPa}$, where it transforms to the $C 2 / c-24$. Hence, we predict that phase III of high-pressure solid hydrogen is polymorphic.
\end{abstract}

DOI: 10.1103/PhysRevB.100.155103

\section{INTRODUCTION}

The phase diagram of high-pressure hydrogen is a challenging problem in condensed matter and high-pressure physics. It has been extensively studied since 1935 [1] by experiment, theory, and more recently computational methods. The main interests are the relevance of solid metallic hydrogen to room-temperature superconductivity [2], possible existence of a metallic liquid ground state [3,4], and astrophysics [5-7].

In this work, we focus on low-temperature phases of solid hydrogen. Infrared (IR) and Raman measurements suggest the existence of several phases and phase transitions within the low-temperature region of the phase diagram. Phase I, which is stable up to $110 \pm 5 \mathrm{GPa}$, is a molecular solid composed of quantum rotors arranged in a hexagonal closepacked structure. Changes in the low-frequency regions of the Raman and IR spectra imply the existence of phase II, also known as the broken-symmetry phase, above $110 \pm 5 \mathrm{GPa}$. The appearance of phase III at $\sim 150 \mathrm{GPa}$ is verified by a large discontinuity in the Raman spectrum and a strong rise in the spectral weight of the molecular vibrons [5]. The IR activity increases dramatically upon transition from phase II to phase III, and the IR and Raman vibron frequencies soften by about $80(1 / \mathrm{cm})$ [8]. Phase IV, characterized by

\footnotetext{
*sam.azadi@kcl.ac.uk
}

two vibrons in its Raman spectrum, was discovered at $300 \mathrm{~K}$ and pressures above $230 \mathrm{GPa}$ [9-11]. A new phase has been observed at pressures above $200 \mathrm{GPa}$ and higher temperatures (for example, $480 \mathrm{~K}$ at $255 \mathrm{GPa}$ ) [12]. This phase is thought to meet phases I and IV at a triple point, near which hydrogen retains its molecular character. The most recent experimental results [13] indicate that $\mathrm{H}_{2}$ and hydrogen deuteride at $300 \mathrm{~K}$ and pressures greater than $325 \mathrm{GPa}$ transform to a new phase $\mathrm{V}$, characterized by substantial weakening of the vibrational Raman activity. The structure of high-pressure solid hydrogen above $150 \mathrm{GPa}$ is experimentally unknown. Thus, theoretical and computational techniques play a crucial role in determining the structure of solid hydrogen. The main goal of this work is to determine phase III of solid hydrogen using the most accurate $a b$ initio techniques.

The electronic structure properties and lattice dynamic of solid hydrogen were mainly investigated using density functional theory (DFT) with local and semilocal exchangecorrelation (XC) functionals [14-24] including van der Waals $\mathrm{XC}$ functionals [25]. In particular, DFT with generalized gradient approximation (GGA) functionals were widely applied to search for low-energy crystal candidate structures and to calculate their vibrational properties. More accurate results for the phase diagram [26-28], excitonic, and quasiparticle band gaps of insulator phases $[29,30]$ were obtained by many-body wave function-based quantum Monte Carlo (QMC) methods [31-33]. These QMC results were used for benchmarking DFT functionals, and it was indicated that the GGA for the XC 
functional can dramatically alter the predicted phase diagram [21].

The crystal structure is the fundamental input for the firstprinciple calculations. Due to lack of established experimental results for the crystal structure, there is no option but to use the structures predicted by DFT. Most of the structures have been predicted using the Perdew-Burke-Ernzerhof (PBE) [34] XC functional $[14,15]$. Yet it is now widely accepted that DFT results for high-pressure hydrogen strongly depends on the choice of employed XC functional $[20,24]$. To the best of our knowledge, there is no comparison between the predicted structures by DFT-based structure prediction methods, which are performed using different XC functionals. The QMC results rely on the structures that are obtained by DFT simulations since structure prediction by QMC is yet unaffordable due to computational costs of dealing with many-body wave functions. Therefore, benchmarking DFT functionals and calculating the phase diagram using different $\mathrm{XC}$ functionals are important for finding the most accurate $\mathrm{XC}$ functional. Moreover, the functionality of the enhancement factor within the high-density regime and treatment of the exchange energy in DFT functionals yield different phase stability and phase transitions. Fortunately, there are some properties which are not affected by the XC approximation. For instance, recently we have proposed a rule of thumb of the shorter the molecular bond length, the larger the electronic band gap and the higher the vibron frequencies, which appears to be valid for all considered XC functionals [25].

It was demonstrated that for the enthalpy-pressure (H-P) phase diagram calculations the best performing XC functionals over all densities are the meta-GGA functionals [21]. However, the recently developed strongly constrained and appropriately normed (SCAN) meta-GGA functional [35] has not been applied on solid hydrogen. The behavior of the exchange enhancement factor in the SCAN functional as a function of the density gradient can provide more accurate results than using GGA functionals. Benchmarking DFT functionals indicated that the exchange energy and the exchange enhancement factor play a crucial role in H-P calculations [21]. In this work, we calculate the H-P phase diagram of molecular structures using the SCAN XC functional.

The derivative discontinuity $\Delta_{\mathrm{XC}}$ of the DFT-XC functional differs from the single-particle Kohn-Sham energy band gap. Local and semilocal DFT XC functionals underestimate the fundamental gap because $\Delta_{\mathrm{XC}}=0$ for them. The inclusion of Hartree-Fock exchange enables us to approximate the exchange contribution of $\Delta_{\mathrm{XC}}$, but owing to the nonlocality, the exchange energy overestimates the band gap. Therefore, hybrid DFT, which includes a fraction of HartreeFock exchange, usually yields an improved description of the electronic structure of insulators. Moreover, because of the absence of an artificial self-repulsion between the occupied states, Hartree-Fock exchange cancels the self-interaction contribution of the Hartree energy and consequently provides a more accurate method to calculate the Kohn-Sham spectra of insulators than GGA. The fraction of Hartree-Fock exchange used in hybrid DFT can be considered as a variational parameters. In this work we optimize this variational parameter using the diffusion Monte Carlo (DMC) method to build an efficient variational many-body wave function, which accurately describes the electronic structural properties of insulators phases.

\section{COMPUTATIONAL DETAILS}

In this work, we consider the molecular structures of $C 2 / c-24, P 6_{3} / m-16, P c-48, P b c n-48$, Cmca-24, Cmca-8, $P 6_{1} 22-36, P_{c a 2}-48, P n a 2_{1}-48, C 2-48$, and $P 2_{1} / c-8$ within the pressure range of 100 to $500 \mathrm{GPa}$. The number after the hyphen indicates the number of hydrogen atoms in the primitive cell used in our calculations. Our DFT calculations were carried out using the latest version of the QuantumEspresso suite of programs [36]. We used a basis set of plane waves with an energy cutoff of $100 \mathrm{Ry}$. For the geometry and cell optimizations a $16 \times 16 \times 16 \mathbf{k}$-point mesh is employed for all the structures except of Cmca-8 and $P 2_{1} / c-8$ for which a $24 \times 24 \times 24 \mathbf{k}$-point mesh is used. The quasi-Newton algorithm was used for all cell and geometry optimization, with a convergence thresholds on the total energy and forces of $0.01 \mathrm{mRy}$ and $0.1 \mathrm{mRy} / \mathrm{Bohr}$, respectively. To calculate the SCAN enthalpy-pressure phase diagram, we used a normconserving Troullier-Martin pseudopotential without nonlinear core corrections, which was generated by the SCAN XC functional [37]. It was demonstrated that use of other conventional pseudopotentials, which are not explicitly produced for the SCAN functional, can lead to discrepancies for some systems [37]. The relative enthalpy-pressure phase diagram is simulated by fitting a quartic polynomial with five fitting parameters on nine enthalpy-pressure DFT points within the pressure range of 100 to $500 \mathrm{GPa}$.

All QMC calculations were performed via the CASINO package using a Slater-Jastrow trial wave function [38]. The orbitals of the Slater determinant were obtained by means of DFT within the local density approximation using the developer version of the Quantum-Espresso code. The Slater orbitals were generated using a norm-conserving pseudopotential in conjunction with a basis set energy cutoff of 400 Ry that were transformed into a localized blip polynomial basis [39]. The PBE1 exchange-correlation functional, in which the mixing of exact-exchange parameter $\alpha=10 \%, 25 \%, 40 \%, 60 \%$, and $80 \%$, was used to optimize the atomic coordinates and generate the single-particle Kohn-Sham orbitals to be used in the QMC calculations. For the geometry optimization, the PBE pseudopotential is used. The DMC results were obtained using a real $\Gamma$-point wave function, $2 \times 2 \times 2$ super cell size, and time step of 0.005 a.u. We used the the model-periodic Coulomb interactions [40] to correct for Coulomb finite-size errors. We used the conventional Jastrow factor that includes the polynomial onebody electron-nucleus, two-body electron-electron, and threebody electron-electron-nucleus terms, which were optimized by minimizing the variance at the VMC level [41].

\section{RESULTS AND DISCUSSION}

\section{A. SCAN phase diagram}

In the following, we first discuss the predicted DFT-SCAN phase diagram. Figure 1 illustrates the H-P phase diagram for the molecular structures of solid hydrogen within the pressure range of 100 to $500 \mathrm{GPa}$, which is simulated using 


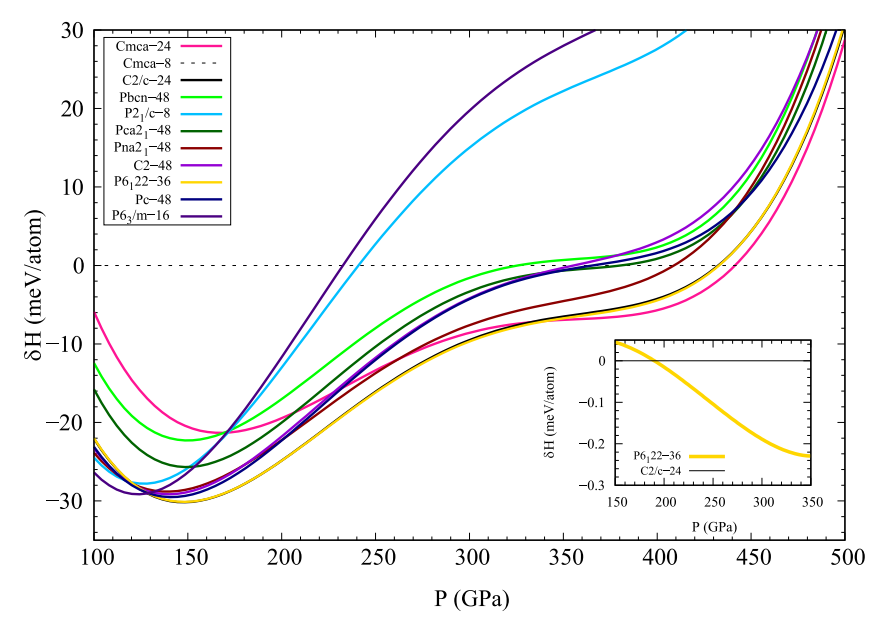

FIG. 1. The static relative enthalpy-pressure phase diagram for solid molecular hydrogen as calculated by the SCAN XC functional. There are four phase transitions: $P 6_{3} / m-16$ to $C 2 / c-24$ at $129 \mathrm{GPa}$, $C 2 / c$-24 to $P 6_{1} 22-36$ at $190 \mathrm{GPa}, P 6_{1} 22-36$ to $C m c a-24$ at $343 \mathrm{GPa}$, and $\mathrm{Cmca}-24$ to $\mathrm{Cmca}-8$ at $442 \mathrm{GPa}$. The number after the hyphen indicates the number of hydrogen atoms in the primitive cell used in our DFT calculations.

the SCAN functional. The studied structures were predicted in previous works by ab initio random structure searching method (AIRSS) using GGA functionals at the static level in which the vibrational contributions were not included $[14,15]$. The SCAN H-P phase diagram predicts four phase transitions of hexagonal $P 6_{3} / m-16$ to monoclinic $C 2 / c-24$ at $129 \mathrm{GPa}$, $C 2 / c$-24 to hexagonal $P 6_{1} 22-36$ at $190 \mathrm{GPa}, P 6_{1} 22-36$ to orthorhombic Cmca-24 at $343 \mathrm{GPa}$, and $\mathrm{Cmca}-24$ to $\mathrm{Cmca}-8$ at $442 \mathrm{GPa}$. The hexagonal $P 6_{3} / m-16$ structure is the most stable phase at pressures below $\sim 130 \mathrm{GPa}$ and is the best candidate for phase I of solid hydrogen. This agrees with low pressure static phase diagram as calculated by GGA functionals $[14,22,24]$. The other candidate for the pressure range below $150 \mathrm{GPa}$ is $P 2_{1} / c$ which was initially predicted with eight atoms per primitive cell. Previous static DFT calculations using different GGA functionals, where the $P 6_{3} / m-16$ were not considered, suggested that the $P 2_{1} / c-24$ with 24 hydrogen atoms per primitive cell is the best candidate for phase I [28].

According to the SCAN phase diagram, $C 2 / c-24$ is stable within the pressure window of 129-190 GPa, and it transforms to $P 6_{1} 22-36$ at $190 \mathrm{GPa}$. The $P 6_{1} 22-36$ phase has been predicted by AIRSS with the Becke-Lee-Yang-Parr (BLYP) [42] XC functional [43]. According to the static DFT-BLYP phase diagram the $C 2 / c-24$ is more stable than the $P 6_{1} 22-36$ phase within pressure range of $100-350 \mathrm{GPa}$, whereas the $P 6_{1}$ 22-36 phase stabilizes because of the zero point energy (ZPE) contributions. The DFT-BLYP Gibbs free energy phase diagram at zero temperature, where the ZPE is taken into account, suggested that $P 6_{1} 22-36$ is more stable than the $C 2 / c-24$ phase at pressures below $180 \mathrm{GPa}$ [43]. Our static SCAN phase diagram predicts that the $P 6_{1} 22-36$ structure is the best candidate for phase III of solid hydrogen above $\sim 200 \mathrm{GPa}$ and below $\sim 340 \mathrm{GPa}$. The Cmca-24 phase is stable from $343 \mathrm{GPa}$ till it transforms to Cmca-8 at $442 \mathrm{GPa}$. Our DMC calculations indicated a very similar phase transition of
Cmca-24 to Cmca-8 at $439 \mathrm{GPa}$ [27]. All previous studies of the high-pressure solid hydrogen phase diagram, which were carried out using DFT-based methods, predict that solid hydrogen adopts the Cmca symmetry before atomization. Taking into account the consistent prediction of previous first-principles calculations within different levels of theory, we propose that low-temperature solid hydrogen adopts a Cmca symmetry before atomization, which is independent of particular XC functional (Fig. 2).

We studied the recently predicted structures of $P c a 2_{1}-48$ and Pna $2_{1}-48$, which have been suggested as the best candidates for phase $\mathrm{V}$ at pressures above $300 \mathrm{GPa}$ [44]. We found that none of these structure are stable at any pressure range. According to the BLYP phase diagram, at a pressure above $300 \mathrm{GPa}$ [44], $C 2 / c$ is stable until it transforms into the $\mathrm{Cmca}-12$ phase at pressures above $\sim 420 \mathrm{GPa}$. The BLYP Gibbs free energy calculations predict that at low and room temperatures the $C 2 / c$ structure transforms into $\mathrm{Cmca}-12$ at around $350 \mathrm{GPa}$. The static SCAN phase diagram indicates a phase transition around $343 \mathrm{GPa}$, namely, the $P 6_{1} 22$ to Cmca-24 phase transition. The BLYP dynamic phase diagram at zero temperature, where the ZPE contributions are included, showed the $P 6_{3} / \mathrm{m}$ to $C 2 / \mathrm{c}$ phase transition at $130 \mathrm{GPa}$, which is close to the prediction of the static SCAN phase diagram. The reason could be due to the fact that the BLYP-ZPE difference between the $P 6_{3} / m$ and $C 2 / c$ phases within the pressure range of $110-150 \mathrm{GPa}$ is smaller than $3 \mathrm{meV} /$ atom [24].

\section{B. Diffusion Monte Carlo phase diagram}

Within the studied pressure range, the SCAN enthalpy difference between the $C 2 / c$ and $P 6_{1} 22$ phases is less than $1 \mathrm{meV} /$ atom, which is below the accuracy of DFT. The DFT energy difference between these two phases within the pressure window of 160-260 GPa is around $0.3 \mathrm{meV} /$ atom, which is affected by ZPE and thermal contributions. The IR and Raman spectra of $C 2 / c$ and $P 6_{1} 22$ were calculated using DFT [43]. Their results indicate that, since the $C 2 / c$ and $P 6_{1} 22$ structures are identical, the frequencies of the active modes are indistinguishable and agree with available experimental data. The only important difference between the two signals is the stronger IR vibron peak for the $P 6_{1} 22$ phase, which agrees with the experimental report that in phase III the IR activity is much larger than that in phase II. The main conclusion is that the IR and Raman spectra of $C 2 / c$ and $P 6_{1} 22$ are consistent with the corresponding spectra observed for phase III, which is why the structure of phase III cannot be determined purely based on its vibrational response. Hence, we apply a higher level of theory to determine phase III of solid hydrogen.

In the rest of this work, we focus on only two structures of $C 2 / c(C 2 / c-24)$ and $P 6_{1} 22\left(P 6_{1} 22-36\right)$, which are the most likely candidates for phase III within the pressure range below $300 \mathrm{GPa}$ and above $150 \mathrm{GPa}$. To calculate the phase transition between these two candidates and determining the structure of phase III, we employed the DMC method. Phase III of solid hydrogen has a finite energy band gap below $300 \mathrm{GPa}$, and the phase transition between the $C 2 / c$ and $P 6_{1} 22$ structures is a pressure driven insulator-insulator structural transition. For the DMC simulations, our initial aim is to find the trial 

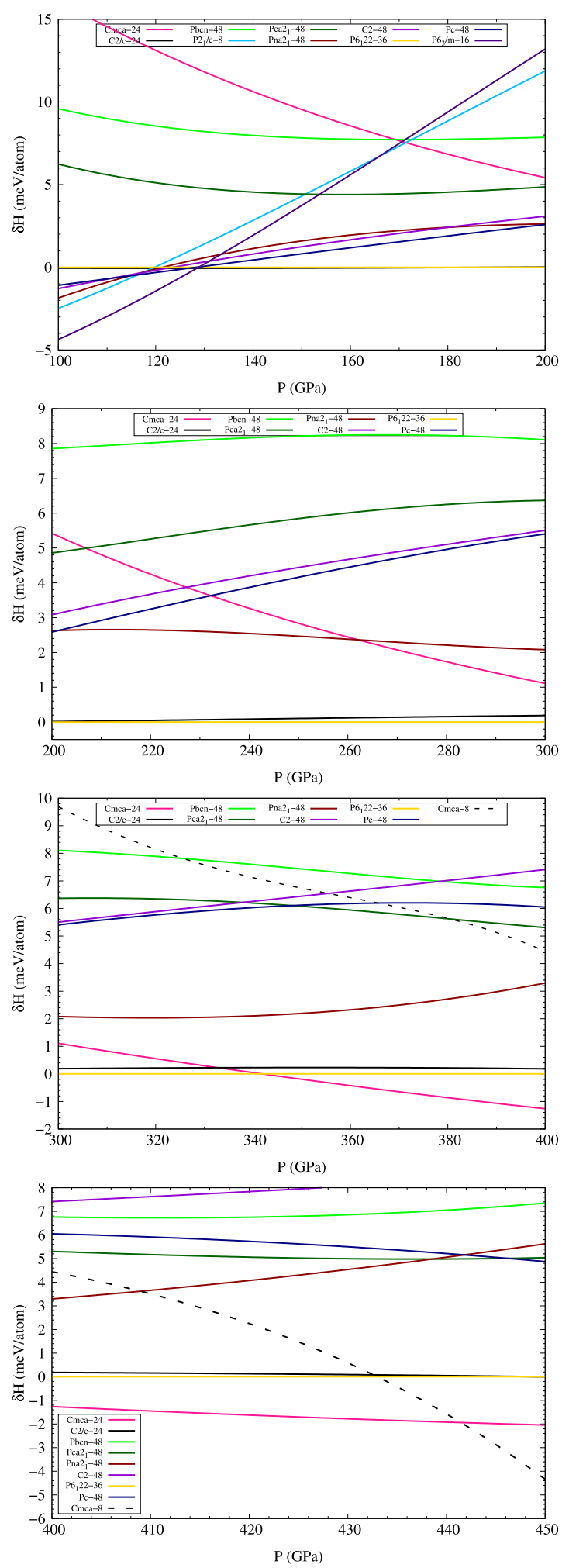

FIG. 2. The static relative enthalpy-pressure phase diagram of solid molecular hydrogen. The phase diagram is separated into four pressure ranges of 100-200, 200-300, 300-400, and 400-450 GPa, respectively. The reference zero line corresponds to the $P 6_{1} 22-36$ phase. The energy difference between the $P 6_{1} 22-36$ and $C 2 / c-24$ structures is less than $1 \mathrm{meV} /$ atom. The observed phase transitions are $P 6_{3} / m-16$ to $C 2 / c-24$ at $129 \mathrm{GPa}, C 2 / c-24$ to $P 6_{1} 22-36$ at $190 \mathrm{GPa}, \mathrm{P6}_{1} 22-36$ to $\mathrm{Cmca}-24$ at $343 \mathrm{GPa}$, and $\mathrm{Cmca}-24$ to $\mathrm{Cmca}-8$ at $442 \mathrm{GPa}$.
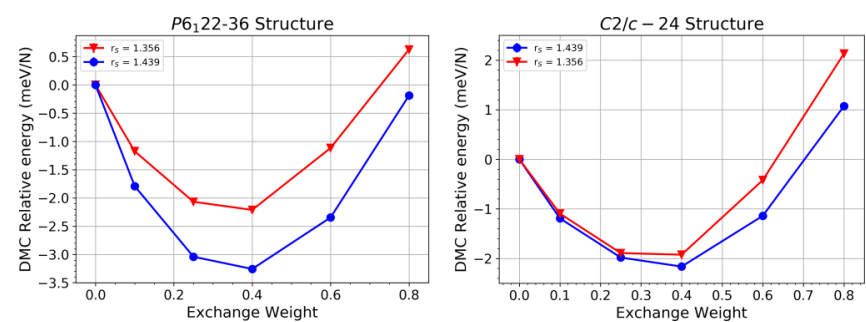

FIG. 3. DMC relative energy as a function of the percentage of exact exchange parameter $\alpha$ in the trial wave function. For $\alpha=0 \%$, our PBE1 XC functional is identical to the PBE functional. The error bars are smaller than $0.1 \mathrm{meV} /$ atom.

many-body wave function that produces the best description of both phases. We generated a set of trail wave functions using the present DFT-PBE1 XC functional, in which the value of the exact-exchange parameter $\alpha$ is varied within the range of 0 to $80 \%$. The DMC approach is a variational method, and therefore the $\alpha$ that gives the lowest ground-state DMC energy provides the most accurate representation of the many-body wave function of the system. We therefore use $\alpha$ to vary the single particle orbitals of the trial wave function. Yet, prior to our DMC calculations, the atomic coordinates of each structure were fully relaxed for each value of $\alpha$.

Figure 3 shows the DMC energies for the $C 2 / c$ and $P 6_{1} 22$ structures as a function of $\alpha$. The reference DMC energy was obtained using the conventional PBE exchange-correlation functional, i.e., $\alpha=0 \%$. The $C 2 / c$ and $P 6_{1} 22$ phases were considered at two different densities with Wigner-Seitz radius of $r_{S}=1.439$ and 1.356 a.u., respectively. For the $P 6_{1} 22$ structure a minimum in the DMC energy is observed for $\alpha=$ $40 \%$. The DMC energy difference between the conventional PBE0 [45,46], where $\alpha=25 \%$, and our PBE1 with $\alpha=40 \%$ at $r_{S}=1.439$ and 1.356 is $0.23(6)$ and $0.24(6) \mathrm{meV} /$ atom. In the case of the $C 2 / c$ structure, this difference at $r_{S}=1.439$ and 1.356 equals $0.18(5)$ and $0.06(5) \mathrm{meV} /$ atom, respectively. The DMC results predict that $\alpha=40 \%$ may tend to produce the best description of the ground-state electronic structure of the $P 6_{1} 22$ and $C 2 / c$ structures, which are the most likely candidates for phase III of solid hydrogen. The energy gain at the minimum for $\alpha=40 \%$ with respect to the PBE XC functional for the $P 6_{1} 22$ phase at $r_{S}=1.439$ and 1.356 is $-3.26(4)$ and $-2.21(3) \mathrm{meV} /$ atom. For the $C 2 / c$ structure, the energy gain at the minimum with respect to PBE at $r_{S}=$ 1.439 and 1.356 equals $-2.16(2)$ and $-1.92(3) \mathrm{meV} /$ atom, respectively. Our DMC results indicate that for the $P 6_{1} 22$ and $C 2 / c$ structures, reducing the $r_{S}$, which corresponds to higher densities and consequently larger pressure, reduces the energy gain with respect to the PBE XC functional.

In Table I we have listed the pressure, the nearest-neighbor distance (bond length), and the energy band gap for the $P 6_{1} 22$ and $C 2 / c$ structures, which are obtained using the PBE $(\alpha=$ $0 \%)$ and PBE1 $(\alpha=40 \%)$ XC functionals. The difference between PBE and PBE1 pressures for the $C 2 / c$ and $P 6_{1} 22$ phases at lower density is 3 and $2 \mathrm{GPa}$, respectively. The same pressure difference for the $C 2 / c$ and $P 6_{1} 22$ structures at $r_{S}=1.356$ is 5 and $4 \mathrm{GPa}$. Since the volume is fixed, the pressure difference between PBE and PBE1 functionals is due only to the atomic coordinate optimization. We also used the 
TABLE I. The pressure in GPa, bond length (BL) in $\AA$, and DFT energy band gap in $\mathrm{eV}$ for the $C 2 / c$ and $P 6_{1} 22$ structures, which are obtained at two densities of $r_{S}=1.439$ and $r_{S}=1.356$ using the $\operatorname{PBE}(\alpha=0 \%)$ and PBE1 ( $\alpha=40 \%)$ Xc functionals, respectively.

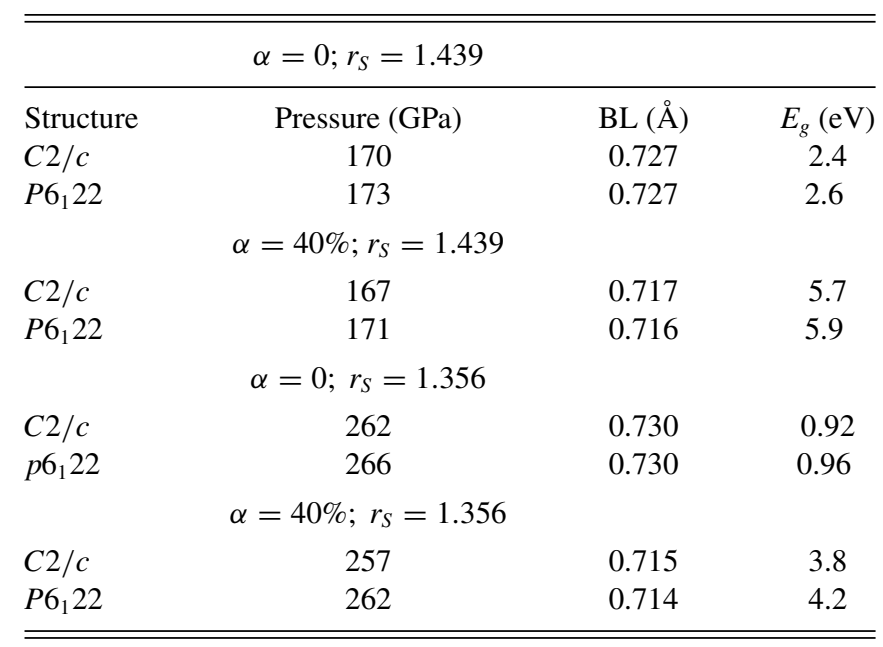

PBE1 XC functional to calculate the enthalpy of $P 6_{1} 22$ and $C 2 / c$ structures. The electronic structure energy was obtained by DMC, and the $P V$ term was calculated using the PBE1 functional. Figure 4 illustrates the relative DFT and DMC enthalpies of the $C 2 / c$ and $P 6_{1} 22$ structures as a function of exchange weight $\alpha$ with respect to the PBE- $C 2 / c$ enthalpy. Similar to the SCAN prediction, the DFT-enthalpy phase diagrams, which are obtained for different values of $\alpha$, at $r_{S}=$ 1.439 and $r_{S}=1.356$ yield nearly indistinguishable results for the $C 2 / c$ and $P 6_{1} 22$ structures. We found differences between the $C 2 / c$ and $P 6_{1} 22$ phases by using the DMC electronic energy. Since $\alpha$ is not a variational parameter within hybridDFT, increasing the $\alpha$ reduces the Kohn-Sham total electronic energy for the systems with wide energy gaps. Our DMC simulations yields a parabola for the energy as a function of $\alpha$, where the energy gain of the $P 6_{1} 22$ phase at lower densities is larger than that of the $C 2 / c$ one. For both studied phases,
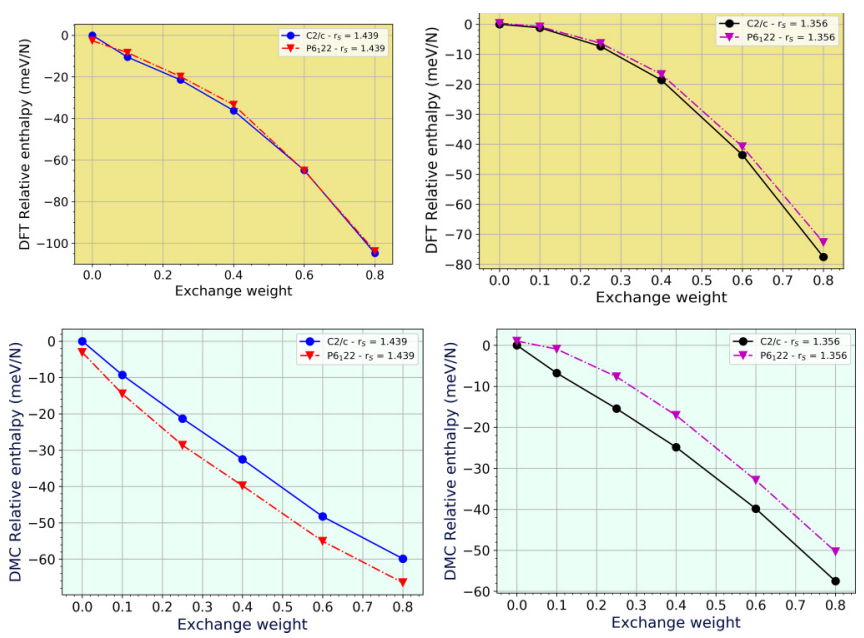

FIG. 4. Relative DFT and DMC enthalpies of $C 2 / c$ and $P 6_{1} 22$ structures as function of exchange weight. The reference point at each $r_{S}$ is the enthalpy of $C 2 / c$ phase. the DMC enthalpy reduces with $\alpha$ due to contributions of the dominating DFT- $P V$ term, which is also decreasing with $\alpha$.

Figure 5 illustrates the relative enthalpy of $P 6_{1} 22$ with respect to the $C 2 / c$ structure. Our DMC H-P diagrams indicate that $P 6_{1} 22$ is the stable phase within pressure range of 160-210 GPa and that it transforms to the $C 2 / c$ structure at around $210-220 \mathrm{GPa}$. The $C 2 / c$ structure is the best candidate for phase III at pressures above $210 \mathrm{GPa}$, whereas the $P 6_{1} 22$ is the most likely candidate for phase III below $210 \mathrm{GPa}$. Our DMC phase diagram prediction is based on the DFT pressure, which introduces a $\sim 3-4 \mathrm{meV} /$ atom error within the enthalpy [26]. Obviously, using the DMC pressure for the static enthalpy-pressure phase diagram may alter the predicted outcomes. The DMC phase diagram indicates that phase III of solid hydrogen could be polymorphic. Our prediction on the polymorphic nature of phase III agrees with recent nuclear magnetic resonance spectroscopy observations [47], where the quantitative results are strongly depend on the used XC functional. A very recent experimental observation shows a first-order phase transition near $425 \mathrm{GPa}$ from an insulating molecular phase to metallic hydrogen [48]. The synchrotron IR spectroscopy measurement indicates the stability of the insulating $C 2 / c-24$ phase in the high-density regime before matallization. Our DMC results for phase III and the stability of $P 6_{1} 22$ at low density and $C 2 / c$ at higher pressures agrees with this recent experiment.

Including the DFT-based ZPE contribution in our phase diagram calculations changes the phase stability of the phases and the transitions between them. We expect that including the phonon energies will also change the SCAN H-P phase diagram. Note that the lattice dynamic should also be calculated using the SCAN functional, for instance, by means of linear response theory or using the direct atom displacement method. The Gibbs free energy calculations up to room temperature for the $P 6_{1} 22$ and $C 2 / c$ structures, which were performed by DFT-PBE and DFT-BLYP [43], indicate that the relative phonon free energy within the pressure range of 150-300 GPa, in which the $P 6_{1} 22$ to $C 2 / c$ phase transition occurs, is less than $2 \mathrm{meV} /$ atom. Based on our DMC enthalpy pressure phase diagram, the energy difference between the

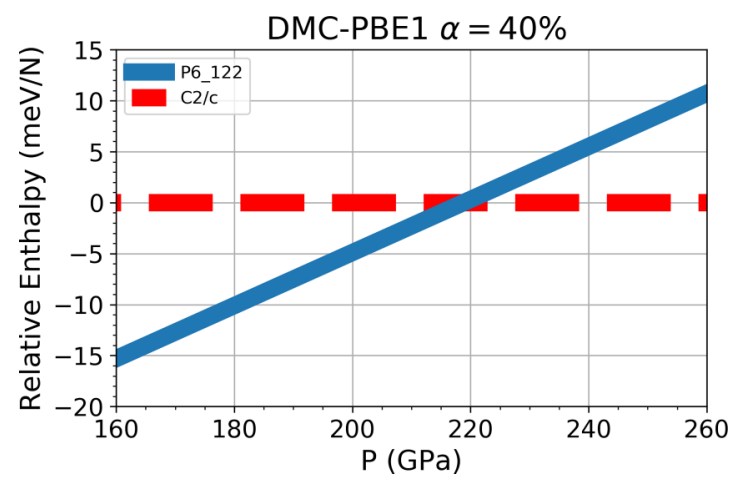

FIG. 5. Relative stability of $P 6_{1} 22$ with respect to the $C 2 / c$ structure. The enthalpy is obtained using the DMC energy and the DFT- $P V$ term at $\alpha=40 \%$. The $P 6_{1} 22$ phase transforms into $C 2 / c$ at a pressure of around $210-220 \mathrm{GPa}$. The widths of the H-P lines indicates the estimated uncertainties in the enthalpy calculations, which is about $\sim 3 \mathrm{meV} /$ atom. 
$P 6_{1} / 22$ and $C 2 / c$ phases at 160 and $200 \mathrm{GPa}$ is $-15(3)$ and -5 (3) $\mathrm{meV} /$ atom, respectively. Therefore, we expect that the effects of including the ZPE and thermal contributions on the DMC H-P phase diagram are negligible.

\section{CONCLUSION}

In the first part of this work, we employed the SCAN meta-GGA XC functional to provide a revised static enthalpypressure phase diagram for 11 competitive molecular structures of solid hydrogen within the pressure range of 100-500 GPa. Our SCAN enthalpy-pressure phase diagram predicts four phase transitions of hexagonal $P 6_{3} / \mathrm{m}-16$ to monoclinic $C 2 / c-24$ at $129 \mathrm{GPa}, C 2 / c-24$ to hexagonal $P 6_{1}$ 22-36 at $190 \mathrm{GPa}, \mathrm{P}_{1} 22-36$ to orthorhombic $\mathrm{Cmca}-24$ at $343 \mathrm{GPa}$, and $\mathrm{Cmca}-24$ to $\mathrm{Cmca}-8$ at $442 \mathrm{GPa}$. Moreover, we compared all the available phase diagrams for high-pressure solid hydrogen, which were obtained by different DFT XC approximations and quantum Monte Carlo-based methods, and proposed a new rule of thumb that the molecular solid high-pressure hydrogen obeys Cmca symmetry before dissociating into an atomic phase. We previously proposed our first rule of thumb about insulating molecular structures of high-pressure solid hydrogen [25], which is the shorter the molecular bond length the larger the electronic band gap and the higher vibron frequencies.
In the second part of this work, we focused on determining the insulating phase III using the many-body wave function-based DMC method. We considered two competitive candidates, namely, the $C 2 / c$ and $P 6_{1} 22$ structures that have finite energy band gaps. To find the most accurate trial manybody wave function, we took into account the fraction of exact exchange $\alpha$ as a variational parameter, which was then optimized by DMC. We found that $\alpha=40 \%$ is the optimized value that provides the lowest ground-state electronic structure total energy. The energy gain with respect to the conventional PBE XC functional, where $\alpha=0 \%$, depends on the atomic structure and density. Our DMC enthalpy-pressure phase diagram indicates that phase III of solid hydrogen adopts two structures, the one $P 6_{1} 22$, which is stable below $\sim 210 \mathrm{GPa}$, and the $C 2 / c$ phase, which is stable at pressures above $\sim 210 \mathrm{GPa}$.

\section{ACKNOWLEDGMENTS}

This project has received funding from the European Research Council (ERC) under the European Union's Horizon 2020 research and innovation programme (Grant Agreement No. 716142). The generous allocation of computing time by the Paderborn Center for Parallel Computing $\left(\mathrm{PC}^{2}\right)$ on OCuLUS and the FPGA-based supercomputer NOCTUA is kindly acknowledged.
[1] E. Wigner and H. B. Huntington, J. Chem. Phys. 3, 764 (1935).

[2] N. W. Ashcroft, Phys. Rev. Lett. 21, 1748 (1968).

[3] S. A. Bonev, E. Schwegler, T. Ogitsu, and G. Galli, Nature (London) 431, 669 (2004).

[4] J. Chen, X.-Z. Li, Q. Zhang, M. I. J. Probert, C. J. Pickard, R. J. Needs, A. Michaelides, and E. Wang, Nat. Commun. 4, 2064 (2013).

[5] H. K. Mao and R. J. Hemley, Rev. Mod. Phys. 66, 671 (1994).

[6] V. L. Ginzburg, Phys. Usp. 42, 353 (1999).

[7] J. M. McMahon, M. A. Morales, C. Pierleoni, and D. M. Ceperley, Rev. Mod. Phys. 84, 1607 (2012).

[8] X.-Z. Li, B. Walker, M. I. J. Probert, C. J. Pickard, R. J. Needs, and A. Michaelides, J. Phys.: Condens. Matter 25, 085402 (2013).

[9] M. I. Eremets and I. A. Troyan, Nat. Mater. 10, 927 (2011).

[10] R. T. Howie, C. L. Guillaume, T. Scheler, A. F. Goncharov, and E. Gregoryanz, Phys. Rev. Lett. 108, 125501 (2012).

[11] R. T. Howie, I. B. Magdău, A. F. Goncharov, G. J. Ackland, and E. Gregoryanz, Phys. Rev. Lett. 113, 175501 (2014).

[12] R. T. Howie, P. Dalladay-Simpson, and E. Gregoryanz, Nat. Mater. 14, 495 (2015).

[13] P. Dalladay-Simpson, R. T. Howie, and E. Gregoryanz, Nature (London) 529, 63 (2016).

[14] C. J. Pickard and R. J. Needs, Nat. Phys. 3, 473 (2007).

[15] C. J. Pickard, M. Martinez-Canales, and R. J. Needs, Phys. Rev. B 85, 214114 (2012).

[16] A. F. Goncharov, J. S. Tse, H. Wang, J. Yang, V. V. Struzhkin, R. T. Howie, and E. Gregoryanz, Phys. Rev. B 87, 024101 (2013).
[17] I. B. Magdău and G. J. Ackland, Phys. Rev. B 87, 174110 (2013).

[18] I. I. Naumov, R. E. Cohen, and R. J. Hemley, Phys. Rev. B 88, 045125 (2013).

[19] M. A. Morales, J. M. McMahon, C. Pierleoni, and D. M. Ceperley, Phys. Rev. B 87, 184107 (2013).

[20] R. C. Clay, J. Mcminis, J. M. McMahon, C. Pierleoni, D. M. Ceperley, and M. A. Morales, Phys. Rev. B 89, 184106 (2014).

[21] R. C. Clay, M. Holzmann, D. M. Ceperley, and M. A. Morales, Phys. Rev. B 93, 035121 (2016).

[22] S. Azadi and T. D. Kühne, JETP Lett. 95, 449 (2012).

[23] R. Singh, S. Azadi, and T. D. Kühne, Phys. Rev. B 90, 014110 (2014).

[24] S. Azadi and W. M. C. Foulkes, Phys. Rev. B 88, 014115 (2013).

[25] S. Azadi and G. J. Ackland, Phys. Chem. Chem. Phys. 19, 21829 (2017).

[26] S. Azadi, W. M. C. Foulkes, and T. D. Kühne, New J. Phys. 15, 113005 (2013).

[27] S. Azadi, B. Monserrat, W. M. C. Foulkes, and R. J. Needs, Phys. Rev. Lett. 112, 165501 (2014).

[28] N. D. Drummond, B. Monserrat, J. H. Lloyd-Williams, P. López Ríos, C. J. Pickard, and R. J. Needs, Nat. Comm. 6, 7794 (2015).

[29] S. Azadi, N. D. Drummond, and W. M. C. Foulkes, Phys. Rev. B 95, 035142 (2017).

[30] S. Azadi, R. Singh, and T. D. Kühne, J. Comp. Chem. 39, 262 (2018).

[31] W. M. C. Foulkes, L. Mitas, R. J. Needs, and G. Rajagopal, Rev. Mod. Phys. 73, 33 (2001). 
[32] S. Azadi and W. M. C. Foulkes, J. Chem. Phys. 143, 102807 (2015).

[33] S. Azadi and R. E. Cohen, J. Chem. Phys. 143, 104301 (2015).

[34] J. P. Perdew, K. Burke, and M. Ernzerhof, Phys. Rev. Lett. 77, 3865 (1996).

[35] J. Sun, A. Ruzsinszky, and J. P. Perdew, Phys. Rev. Lett. 115, 036402 (2015).

[36] P. Giannozzi, S. Baroni, N. Bonini, M. Calandra, R. Car, C. Cavazzoni, D. Ceresoli, G. L. Chiarotti, M. Cococcioni, I. Dabo et al., J. Phys.: Condens. Matter 21, 395502 (2009).

[37] Y. Yao and Y. Kanai, J. Chem. Phys. 146, 224105 (2017).

[38] R. J. Needs, M. D. Towler, N. D. Drummond, and P. López Ríos, J. Phys.: Condens. Matter 22, 023201 (2010).

[39] D. Alfé and M. J. Gillan, Phys. Rev. B 70, 161101(R) (2004).

[40] L. M. Fraser, W. M. C. Foulkes, G. Rajagopal, R. J. Needs, S. D. Kenny, and A. J. Williamson, Phys. Rev. B 53, 1814 (1996).
[41] N. D. Drummond and R. J. Needs, Phys. Rev. B 72, 085124 (2005).

[42] C. Lee, W. Yang, and R. G. Parr, Phys. Rev. B 37, 785 (1988).

[43] B. Monserrat, R. J. Needs, E. Gregoryanz, and C. J. Pickard, Phys. Rev. B 94, 134101 (2016).

[44] B. Monserrat, N. D. Drummond, P. Dalladay-Simpson, R. T. Howie, P. Lòpez Riòs, E. Gregoryanz, C. J. Pickard, and R. J. Needs, Phys. Rev. Lett. 120, 255701 (2018).

[45] J. P. Perdew, M. Ernzerhof, and K. Burke, J. Chem. Phys. 105, 9982 (1996).

[46] A. D. Becke, J. Chem. Phys. 98, 1372 (1993); 98, 5648 (1993); 104, 1040 (1996).

[47] B. Monserrat, S. E. Ashbrook, and C. J. Pickard, Phys. Rev. Lett. 122, 135501 (2019).

[48] P. Loubeyre, F. Occelli, and P. Dumas, arXiv:1906.05634 (2019). 